\title{
Interação de proteínas Cry1 e Vip3A de Bacillus thuringiensis para controle de lepidópteros-praga
}

\author{
Paula Cristina Brunini Crialesi-Legori(1), Camila Chiaradia Davolos ${ }^{(1)}$, Ana Rita Nunes Lemes ${ }^{(1)}$, \\ Suzana Cristina Marucci(1), Manoel Victor Franco Lemos ${ }^{(1)}$, Odair Aparecido Fernandes ${ }^{(2)}$ \\ e Janete Apparecida Desidério(1)
}

\begin{abstract}
(1)Universidade Estadual Paulista (Unesp), Departamento de Biologia Aplicada à Agropecuária, Rodovia Prof. Paulo Donato Castellane, Km 5, CEP 14884-900 Jaboticabal, SP, Brasil. E-mail: pbcrialesi@yahoo.com.br, camila.davolos@yahoo.com.br, anarita_lemes@yahoo.com.br, suzy_marucci@yahoo.com.br, mvictor@fcav.unesp.br, janete@fcav.unesp.br (2)Unesp, Departamento de Fitossanidade. E-mail: oafernandes@fcav.unesp.br
\end{abstract}

Resumo - O objetivo deste trabalho foi avaliar a suscetibilidade das lagartas Anticarsia gemmatalis (Lepidoptera: Erebidae) e Chrysodeixis includens (Lepidoptera: Noctuidae) às proteínas Cry1 e Vip3A, bem como determinar se há a interação entre essas proteínas no controle das duas espécies. Bioensaios com as proteínas isoladas e em combinações foram realizados, e as concentrações letais $\mathrm{CL}_{50}$ e $\mathrm{CL}_{90}$ foram estimadas para cada condição. As proteínas Cry1Aa, Cry1Ac e Vip3Af foram as mais efetivas no controle de A. gemmatalis, enquanto Cry1Ac, Vip3Aa e Vip3Af foram mais efetivas no de C. includens. As proteínas Cry1Ac e Cry1Ca causaram maior inibição do desenvolvimento das larvas sobreviventes à $\mathrm{CL}_{50}$, em ambas as espécies. Combinações entre Vip3A e Cry1 apresentam efeito sinérgico no controle das espécies e a combinação Vip3Aa+Cry1Ea destaca-se no controle de $A$. gemmatalis e $C$. includens. Essas proteínas combinadas são promissoras na construção de plantas piramidadas, para o controle simultâneo das pragas.

Termos para indexação: Anticarsia gemmatalis, Chrysodeixis includens, manejo da resistência, piramidação de genes, sinergismo, soja transgênica.

\section{Interaction of Cry1 and Vip3A proteins of Bacillus thuringiensis for the control of lepidopteran insect pests}

\begin{abstract}
The objective of this work was to evaluate the susceptibility of Anticarsia gemmatalis (Lepidoptera: Erebidae) and Chrysodeixis includens (Lepidoptera: Noctuidae) caterpillars to Cry1 and Vip3A proteins, as well as to determine if there is any interaction between these proteins on the control of the two species. Bioassays with both isolated and combined proteins were carried out, and lethal concentrations $\mathrm{LC}_{50}$ and $\mathrm{LC}_{90}$ were estimated for each condition. Cry1Aa, Cry1Ac, and Vip3Af were the more effective proteins for the control of A. gemmatalis, while Cry1Ac, Vip3Aa, and Vip3Af were more effective for the control of C. includens. Cry1 Ac and Cry1Ca proteins caused the highest inhibition to the development of larvae that survived the $\mathrm{LC}_{50}$ dose in both species. Different combinations of Vip3A and Cry1 have synergistic effect in the control of both species, and the combination Vip3Aa + Cry1Ea showed an outstanding control of A. gemmatalis and C. includens. These proteins are promising for building pyramided plants for the simultaneous control of the pests.
\end{abstract}

Index Terms: Anticarsia gemmatalis, Chrysodeixis includens, resistance management, gene pyramiding, synergism, transgenic soybean.

\section{Introdução}

Bacillus thuringiensis (Bt) é uma bactéria Gram-positiva, com atividade inseticida a diversas pragas agrícolas, que pode ser encontrada naturalmente no solo, superfície de plantas, em insetos mortos e em grãos armazenados (Bravo et al., 1998). Em razão de sua atividade entomopatogênica, essa bactéria é amplamente empregada no controle biológico de insetos-praga e pode ser utilizada na forma de bioinseticidas ou em plantas geneticamente modificadas.

Culturas transgênicas que expressam proteínas de B. thuringiensis foram comercializadas pela primeira vez em 1996 (James, 2011) e são comumente utilizadas para o manejo de insetos-praga em todo o mundo. Essas culturas necessitam de menos aplicações de defensivos químicos e permitem redução dos custos de produção, 
especificidade contra as pragas-alvo e menor impacto sobre a biodiversidade (James, 2002). Entretanto, apesar das vantagens, atualmente há grande preocupação com o uso generalizado de culturas Bt, uma vez que elas podem acelerar a seleção de organismos resistentes (Zhang et al., 2013), quando estratégias de manejo adequadas, como adoção de áreas de refúgio, não são utilizadas. $\mathrm{O}$ surgimento da resistência pode estar relacionado à ligação das proteínas inseticidas a receptores no intestino médio das lagartas (Ferré \& Van Rie, 2002). Alternativas para retardar a evolução da resistência têm sido bastante estudadas e misturas de toxinas têm sido consideradas mais eficazes se utilizadas antes da evolução da resistência, mesmo sob pressão de seleção contínua (Xue et al., 2005; Bergamasco et al., 2013).

A expressão dos genes cry de $B$. thuringiensis geralmente ocorre na fase estacionária da célula, com acúmulo do produto na célula-mãe, que é liberada durante a esporulação. Após a ingestão destas células por larvas de insetos suscetíveis e de sua dissolução no lúmen do intestino médio, as proteínas são ativadas e clivadas por proteases, o que leva à produção de fragmentos tóxicos de 60-70 kDa (Bravo et al., 2007), divididos em duas regiões estruturais distintas: a região $\mathrm{N}$-terminal, que contém a toxina ativa; e a região C-terminal.

As proteínas Vip ("vegetative insecticidal proteins") são produzidas e secretadas pelas células bacterianas durante a fase vegetativa e não formam inclusões cristalinas; por esse motivo, e também por não apresentarem homologia de sequência ou estrutural, foram excluídas da nomenclatura das proteínas Cry. As toxinas Vip3 atuam no controle de lepidópteros e, para se tornarem ativas, devem sofrer a clivagem de 199 aminoácidos na região N-terminal (Lee et al., 2003).

Alguns eventos de plantas piramidadas já estão no mercado, como o algodão com tecnologia WideStrike (Dow Agrosciences), obtido por melhoramento clássico entre eventos que contêm os genes crylF e crylAc (Comissão Técnica Nacional de Biossegurança, 2009), e o milho Bt11XMIR162XGA21 (Syngenta Seeds), que possui os genes crylAb, vip3Aa20 e tolerância a herbicida (Comissão Técnica Nacional de Biossegurança, 2010). Recentemente, houve a aprovação da soja transgênica que expressa a proteína
Cry1Ac e tem tolerância ao glifosato, resultado de melhoramento clássico dos eventos MON $87701 \times$ MON 89788, cujo cultivo comercial foi realizado no ano agrícola 2013/2014. Este evento é efetivo principalmente para as espécies A. gemmatalis e C. includens (Bernardi et al., 2012).

Anticarsia gemmatalis Hübner (Lepidoptera: Erebidae) e Chrysodeixis includens Walker (Lepidoptera: Noctuidae) são pragas de importância econômica para a cultura da soja. Atualmente, não há relatos de estudos sobre combinações de toxinas de $B$. thuringiensis para o controle destas espécies.

O objetivo deste trabalho foi avaliar a suscetibilidade das lagartas Anticarsia gemmatalis (Lepidoptera: Erebidae) e Chrysodeixis includens (Lepidoptera: Noctuidae), às proteínas Cry1 e Vip3A, bem como determinar se há a interação entre essas proteínas no controle das duas espécies.

\section{Material e Métodos}

Clones de Escherichia coli XL-1 Blue que continham os genes cryl de $B$. thuringiensis foram obtidos junto ao Plant Research International, Wageningen, Netherlands. Estes clones são portadores dos genes crylAa, crylAb, crylAc, crylCa e crylEa, clonados nos respectivos vetores de expressão pPB08, pPBD140, pB03, pBD150 e pBD160. Os clones de E. coli WK6 que continham os genes de $B$. thuringiensis vip3Aa (National Center for Biotechnology Information, 2013c), vip3Ae (National Center for Biotechnology Information, 2013b) e vip3Af (National Center for Biotechnology Information, 2013a) provieram da empresa Bayer Crop Science (Ghent, Bélgica). Todos os vetores possuíam genes de resistência ao antibiótico ampicilina.

A produção das proteínas Cryl foi realizada de acordo com protocolo descrito por Herrero et al. (2004). Para isso, os clones foram cultivados durante 60 horas, a $28^{\circ} \mathrm{C}$, com agitação de $200 \mathrm{rpm}$, em meio TB ("terrific broth") constituído de: $12 \mathrm{~g}$ de triptona; $24 \mathrm{~g}$ de extrato de levedura; 2,31 g de $\mathrm{KH}_{2} \mathrm{PO}_{4} ; 12,54 \mathrm{~g}$ de $\mathrm{K}_{2} \mathrm{HPO}_{4} ; 4 \mathrm{~mL}$ de glicerol; q.s.p $1.000 \mathrm{~mL}$; além de ampicilina (100 $\left.\mu \mathrm{g} \mathrm{mL}^{-1}\right)$. As células cultivadas foram coletadas por centrifugação por $8 \mathrm{~min}$ a $5.930 \mathrm{~g}$. $\mathrm{O}$ processo de rompimento celular foi realizado em tampão $50 \quad$ mmol $\quad \mathrm{L}^{-1} \quad$ Tris/ $\mathrm{HCl} \quad \mathrm{pH} \quad 8,0$, com $5 \mathrm{mmol} \mathrm{L} \mathrm{L}^{-1}$ EDTA e $100 \mathrm{mmol} \mathrm{L}^{-1} \mathrm{NaCl}$ 
com lisozima (800 $\mu \mathrm{g} \quad \mathrm{g}^{-1}$ de pellet), seguido de lavagens com $20 \mathrm{mmol} \mathrm{\textrm {L } ^ { - 1 }}$ Tris/HCl pH7,5, com $1 \mathrm{~mol} \mathrm{~L}^{-1} \mathrm{NaCl}$ e $1 \%$ de Triton $\times 100$ e solução salina tamponada com fosfato, $10 \mathrm{mmol} \mathrm{\textrm {L } ^ { - 1 }}$ $\mathrm{Na}_{2} \mathrm{HPO}_{4} / \mathrm{KH}_{2} \mathrm{PO}_{4}$ pH 7,4, dissolvidos em $0,8 \%$ (p/v) $\mathrm{NaCl}$. Ambos os procedimentos de lavagem foram realizados em triplicata. Como etapa final, realizou-se a solubilização das proteínas Cry em tampão $50 \mathrm{mmol}^{-1} \mathrm{NaHCO}_{3} \mathrm{pH}$ 10,0, com $100 \mathrm{mmol} \mathrm{L}^{-1} \mathrm{NaCl}$ e $10 \mathrm{mmol} \mathrm{L}^{-1}$ DTT (adicionado imediatamente antes do uso), e incubação por 3 horas à temperatura ambiente, em agitação horizontal. Essa etapa foi seguida de centrifugação por $15 \mathrm{~min}$, a $39.200 \mathrm{~g}$, a $4^{\circ} \mathrm{C}$. O sobrenadante com a pró-toxina solubilizada foi filtrado em membrana de poliestireno de $0,44 \mathrm{~mm}$.

As proteínas Cryl foram utilizadas após solubilização dos corpos de inclusão em tampão (50 mmol L-1 $\mathrm{NaHCO}_{3}$ pH 10,0; $100 \mathrm{mmol} \mathrm{L}^{-1}$ $\mathrm{NaCl} ; 10$ mmol L-1 DTT) conforme Herrero et al. (2004). Para a expressão das proteínas Vip3A, utilizou-se a metodologia descrita por Chakroun et al. (2012), que altera a concentração de isopropil-D-tiogalactopiranosídeo (IPTG) para $1 \mathrm{mmol} \mathrm{L} \mathrm{L}^{-1}$ na indução da expressão. As proteínas foram recuperadas por centrifugação, em tampão fosfato $20 \mathrm{mmol} \mathrm{L}-1\left(\mathrm{NaH}_{2} \mathrm{PO}_{4} \cdot \mathrm{H}_{2} \mathrm{O}\right.$ e $\left.\mathrm{Na}_{2} \mathrm{HPO}_{4} \cdot 2 \mathrm{H}_{2} \mathrm{O}\right)$ pH 7,4, com 0,5 $\mathrm{mol} \mathrm{L}^{-1} \mathrm{NaCl}$.

A expressão de proteínas foi verificada por eletroforese, em gel desnaturante de poliacrilamida a $12 \%$, que continha dodecil sulfato de sódio (SDS-PAGE), conforme Laemmli (1970), com uso de Spectra Multicolor Broad Range Protein Ladder (Fermentas, Vilnius, Lituânia) como marcador de massa molecular, tendo-se seguido as recomendações do fabricante.

A concentração das pró-toxinas Cry1 e Vip3A foi estimada por densitometria em gel de SDS-PAGE, por meio do programa BioNumerics (Applied-Maths), a diferentes concentrações $(0,05$ à $0,8 \mathrm{mg} \mathrm{mL}^{-1}$ ) da proteína soro albumina bovina (BSA), como referência-padrão de massa molecular. Os lisados proteicos solubilizados de cada pró-toxina foram filtrados em $0,22 \mu \mathrm{mol} \mathrm{L} \mathrm{L}^{-1} \mathrm{e}$ armazenados a $-20^{\circ} \mathrm{C}$.

Para a realização dos bioensaios, utilizaram-se os ovos de $A$. gemmatalis e $C$. includens, fornecidos pelo Laboratório de Biologia de Insetos (Esalq/Usp), Piracicaba, SP. Utilizaram-se placas de poliestireno de
128 orifícios $\left(2 \mathrm{~cm}^{2}\right)$ (Cell Wells, Corning Glass Works, Corning, NY, USA) e dieta artificial adaptada conforme Monnerat et al. (2007). Diferentes concentrações de pró-toxinas Cry $1\left(0,1\right.$ a 300,0 $\left.\mathrm{mg} \mathrm{mL}^{-1}\right)$ e Vip3 A (0,5 a $600,0 \mathrm{mg} \mathrm{mL}^{-1}$ ) foram aplicadas à superfície da dieta, em volume de $50 \mu \mathrm{L}$. Em seguida, larvas neonatas foram transferidas individualmente para os poços. Dezesseis larvas foram testadas para cada concentração, tendose realizado o experimento em triplicata (total de 48 larvas por concentração de pró-toxina). Como controle dos ensaios realizados, utilizou-se água destilada, tampão de solubilização das proteínas, lisado proteico de E. coli XL-1 Blue e E. coli WK6 sem o vetor de expressão. Estes componentes não causam mortalidade aos insetos. As placas foram acondicionadas a $25 \pm 2{ }^{\circ} \mathrm{C}$, com umidade relativa de $70 \pm 10 \%$ e fotoperíodo de 14:10 horas (luz:escuro). A avaliação foi realizada após sete dias de alimentação continuada.

As $\mathrm{CL}_{50}$ e $\mathrm{CL}_{90}$ foram estimadas pela análise de Probit (Finney, 1971), com uso do programa Polo-Plus (LeOra Software, Berkeley, CA, USA), em que a $\mathrm{CL}_{50}$ e $\mathrm{CL}_{90}$ correspondem à concentração letal de proteínas responsável pela mortalidade de 50 e $90 \%$ da população, respectivamente. Os intervalos de confiança $(95 \%)$ para $\mathrm{CL}_{50}$ e $\mathrm{CL}_{90}$ foram calculados, e a não sobreposição deste parâmetro foi utilizada para detectar diferenças significativas entre as proteínas. Avaliou-se a inibição do desenvolvimento (ID) das larvas sobreviventes à $\mathrm{CL}_{50}$. Estas larvas foram pesadas em balança analítica com quatro casas decimais e os valores obtidos foram aplicados à fórmula $\mathrm{ID}=100 \times$ (média do peso das larvas da testemunha - média do peso das larvas do tratamento)/ (média do peso das larvas da testemunha), proposta de Huang et al. (2006). A análise de interação das pró-toxinas foi realizada de acordo com a metodologia proposta por Tabashnik (1992), tendo-se utilizado como referência inicial a $\mathrm{CL}_{50}$ estimada previamente para cada uma delas. A mortalidade esperada foi estimada com a fórmula proposta pelo autor como, $\mathrm{CL}_{50}(\mathrm{~m})=1 /\left[\left(\mathrm{pa} / \mathrm{CL}_{50}(\mathrm{a})\right)+\left(\mathrm{pb} / \mathrm{CL}_{50}(\mathrm{~b})\right)\right], \mathrm{em}$ que: $\mathrm{CL}_{50}(\mathrm{~m})$ corresponde à $\mathrm{CL}_{50}$ esperada da mistura de proteínas; $\mathrm{CL}_{50}$ (a) e $\mathrm{CL}_{50}$ (b) são as respectivas concentrações letais para as pró-toxinas individuais; $\mathrm{p}$ significa a proporção de a $(\mathrm{pa})$ e $\mathrm{b}(\mathrm{pb})$ na mistura. A mesma fórmula foi utilizada para determinar a interação das pró-toxinas com os dados obtidos para a 
$\mathrm{CL}_{90} \mathrm{e}$, desta forma, avaliar o fator de sinergismo entre as pró-toxinas testadas para o controle das espécies.

$\mathrm{O}$ fator de sinergismo (FS) serve para indicar quando ocorre a interação sinérgica das pró-toxinas em combinação e é calculado como a razão entre a CL esperada e a CL observada; assim: FS maior do que 1 significa a ocorrência de sinergismo entre as pró-toxinas; FS menor do que 1 representa a interação antagônica; e FS igual a 1 é indicativo de toxicidade aditiva (Wu et al., 1994).

\section{Resultados e Discussão}

A expressão das proteínas Cry1 e Vip3A, analisada por SDS-PAGE, foi confirmada pela visualização de bandas com massa molecular de aproximadamente $135 \mathrm{kDa}$, para as pró-toxinas Cry1, e $88 \mathrm{kDa}$ para as Vip3A (Figura 1). A massa molecular das pró-toxinas Cry está compreendida entre 130 e $135 \mathrm{kDa}$ (Lightwood et al., 2000), enquanto a das pró-toxinas Vip3A é de $88,5 \mathrm{kDa}$ (Loguercio et al., 2002).

Em relação à susceptibilidade de $A$. gemmatalis, Cry1Ac destacou-se das demais pró-toxinas Cry1 e foi, aproximadamente, duas vezes mais tóxica que Cry1Aa, no que se refere aos valores obtidos para $\mathrm{CL}_{50}$ (Tabela 1). Este resultado não foi observado para os valores de $\mathrm{CL}_{90}$, que não diferiram significativamente. Este resultado indica que, em concentrações mais elevadas, ambas as proteínas mostraram-se

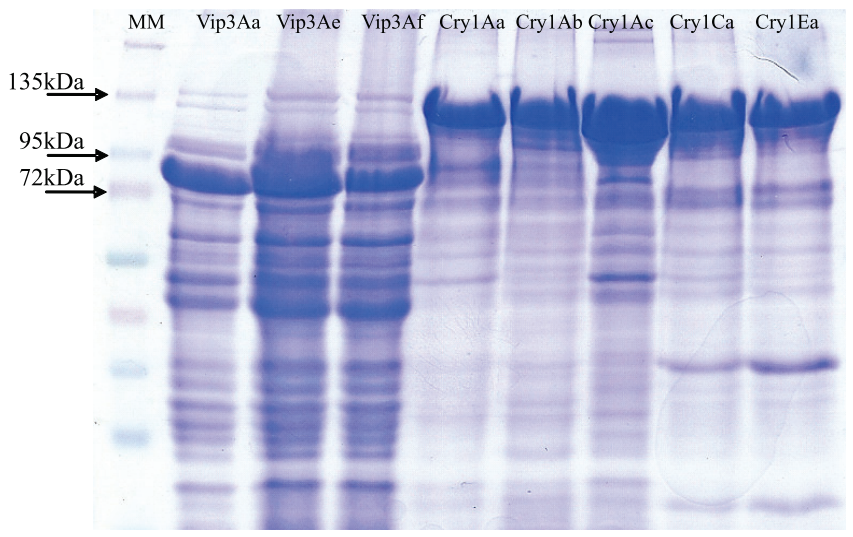

Figura 1. Gel SDS-PAGE do lisado bacteriano de Escherichia coli, com os genes vip3Aa, vip3Ae, vip3Af, crylAa, crylAb, crylAc, crylCa e crylEa. MM, marcador de massa molecular $(\mathrm{kDa})$. igualmente efetivas no controle da espécie (Tabela 1). O presente estudo confirma a eficiência da proteína que está presente na soja transgênica, liberada para comercialização desde 2010. De modo semelhante, em estudo conduzido por Fiuza et al. (2013) para avaliar a suscetibilidade de $A$. gemmatalis a diferentes toxinas Cry, tanto a proteína Cry1Ac quanto a Cry1Aa foram as mais efetivas no controle da praga, enquanto Cry1Ea foi a menos eficiente.

Não houve diferença entre as pró-toxinas Cry1 Ab, Cry1Ca e Cry1Ea quanto ao controle de $A$. gemmatalis, pois houve sobreposição dos valores apresentados nos intervalos de confiança das respectivas $\mathrm{CL}_{50}$ e $\mathrm{CL}_{90}$ obtidas (Tabela 1). Essas proteínas mostraram-se menos efetivas do que Cry1Ac e Cry1Aa no controle da lagarta, nos valores obtidos para $\mathrm{CL}_{90}$. Fiuza et al. (2013) também relataram menor efetividade de Cry1Ea em ensaios de toxicidade com A. gemmatalis. Com base nos valores de $\mathrm{CL}_{90}$, Vip3Af foi a mais eficiente no controle da espécie, tendo sido de sete a oito vezes mais tóxica do que Vip3Aa e Vip3Ae, respectivamente (Tabela 1). Figueiredo et al. (2013) estudaram a suscetibilidade de $A$. gemmatalis à proteína Vip3Aa50 e relataram uma $\mathrm{CL}_{50} 3,8$ vezes inferior à encontrada no presente estudo para Vip3Aa.

Nos ensaios realizados com $C$. includens, observouse que CrylAc, Cry1Ca e Cry1Ea apresentaram maior toxicidade entre as pró-toxinas Cryl testadas

Tabela 1. Suscetibilidade e inibição de desenvolvimento (ID) de larvas neonatas de Anticarsia gemmatalis às proteínas Cry1 e Vip3 de Bacillus thuringiensis.

\begin{tabular}{ccccc}
\hline Proteína & $\mathrm{b} \pm \mathrm{EP}^{(1)}$ & $\mathrm{CL}_{50}(\mathrm{IC} \text { min-max })^{(2)}$ & $\mathrm{ID}(\%)^{(3)}$ & $\mathrm{CL}_{90}(\mathrm{IC} \text { min-max })^{(4)}$ \\
\hline Cry1Aa & $4,5 \pm 0,7$ & $1,8(1,5-2,2)$ & 70 & $3,5(2,9-4,8)$ \\
Cry1Ab & $1,5 \pm 0,4$ & $40(25-107)$ & 40 & $281,4(106-527,6)$ \\
Cry1Ac & $1,6 \pm 0,3$ & $0,75(0,4-1,2)$ & 85 & $4,6(2,4-15,5)$ \\
Cry1Ca & $2,1 \pm 0,3$ & $23,4(16,0-32,4)$ & 90 & $94,8(62,5-196,4)$ \\
Cry1Ea & $2,2 \pm 0,3$ & $52,2(40,0-68,6)$ & 75 & $190,9(129,0-378,3)$ \\
Vip3Aa & $1,1 \pm 0,2$ & $5,4(3,1-9,7)$ & 96 & $72,8(28,9-627,2)$ \\
Vip3Ae & $1,0 \pm 0,2$ & $5,0(2,7-10)$ & 90 & $80,0(29,4-751,4)$ \\
Vip3Af & $2,7 \pm 0,5$ & $3,6(2,6-5,3)$ & 80 & $10,4(6,6-27,7)$ \\
\hline
\end{tabular}

${ }^{(1)}$ Coeficiente angular da reta \pm erro-padrão. ${ }^{(2)} \mathrm{Os}$ valores de $\mathrm{CL}_{50}$ são expressos em ng cm${ }^{-2}$, com $95 \%$ de intervalo de confiança (IC). ${ }^{(3)}$ Inibição do desenvolvimento das larvas alimentadas com as doses de $\mathrm{CL}_{50}{ }^{{ }^{(4)}}$ Os valores de $\mathrm{CL}_{90}$ são expressos em ng cm${ }^{-2}$, com $95 \%$ de intervalo de confiança (IC). Os valores representam a média de três réplicas de 16 larvas por réplica $(\mathrm{n}=48)$. 
(Tabela 2). Em relação à Cry1Aa, as proteínas Cry1Ac, Cry1Ca e Cry1Ea foram de 1,5 a 3,5 vezes mais tóxicas, e Cry1 Ab foi de 2 a 4,5 vezes mais tóxica.

Embora tenha-se destacado das demais proteínas, Cry1Ac foi mais efetiva para $A$. gemmatalis do que para $C$. includens. Estudos realizados com uma linhagem transgênica de soja, que expressava altos níveis de proteína a partir do gene $c r y l A c$, relataram mortalidade total em lagartas de $A$. gemmatalis e redução significativa da população de $C$. includens, em ensaios de suscetibilidade e de alimentação realizados em laboratório (Stewart Junior et al., 1996) e no campo (Walker et al., 2000).

Com base nos valores de $\mathrm{CL}_{90}$, as pró-toxinas Cry1Ac, Cry1Ca e Cry1Ea apresentaram maior toxicidade, e seus intervalos de confiança se sobrepuseram. Walker et al. (2000) relataram que uma linhagem transgênica de soja com altos níveis de proteína a partir do gene sintético crylAc ocasionou mortalidade total em larvas de $A$. gemmatalis e redução significativa da população de $C$. includens, em ensaios de suscetibilidade e de alimentação realizados em laboratório e no campo.

A pró-toxina Vip3Af foi a mais eficiente das Vip3A no controle de $C$. includens, quando foram considerados os valores de $\mathrm{CL}_{50}$ (Tabela 2). Esta eficiência foi aproximadamente 25 e 58 vezes maior do que as apresentadas por Vip3Aa e Vip3Ae, respectivamente. Todavia, de acordo com os valores apresentados na $\mathrm{CL}_{90}$, a toxicidade de Vip3Aa e Vip3Af foi semelhante, pois houve sobreposição dos intervalos de confiança. Yu et al. (1997) relataram que algumas proteínas Vip3 A podem atuar com mais eficiência no controle de pragas cujas proteínas Cry1 não apresentam o melhor controle.

Não houve mortalidade nos insetos mantidos nos controles, que consistiram de água destilada, tampão de solubilização das proteínas ou lisado proteico de E. coli XL-1 Blue e E. coli WK6 sem o vetor de expressão.

As proteínas Cry1Ac e Cry1Ca provocaram ID próximos de $100 \%$ para as duas espécies, quando alimentadas com dietas com $\mathrm{CL}_{50}$ (Tabelas 1 e 2). Em $C$. includens, essa inibição também foi verificada com Cry1 Aa. Nos ensaios com A. gemmatalis, Cry1 Ac foi 20 vezes mais efetiva que Cry $1 \mathrm{Ca}$; porém, quando o desenvolvimento das larvas foi avaliado, verificouse que Cry1Ca reduziu o desenvolvimento das larvas tanto quanto Cry1Ac.
A inibição do desenvolvimento das larvas que sobreviveram à $\mathrm{CL}_{50}$ resultou no aumento do período de desenvolvimento, o que pode levar à redução da população de larvas, em decorrência da maior exposição a inimigos naturais (Ashfaq et al., 2011). As pró-toxinas Vip3A foram eficientes para as duas espécies-alvo do presente estudo, com a maior inibição do desenvolvimento (ID=96\%) obtida com Vip3Aa em A. gemmatalis, e a menor (ID=70\%), com Vip3Af em $C$. includens (Tabelas 1 e 2).

Com base na interação de pró-toxinas Vip3A e Cry1, deduz-se que, das 15 combinações de proteínas avaliadas para cada espécie, somente quatro mostraram-se promissoras para uso no controle biológico de $A$. gemmatalis, e três para o controle de $C$. includens. $\mathrm{O}$ desenvolvimento de resistência de insetos-praga a toxinas Cry foi relatado em relação à proteína Cry1Ab expressa em milho, na África do Sul (Van Rensburg, 2007), Cry1F expressa em milho, em Porto Rico (Storer et al., 2010), e Cry1Ac em algodão, na Índia (Dhurua \& Gujar, 2011). Assim, a combinação de proteínas inseticidas é uma alternativa eficiente para o manejo de resistência, visto que combinações efetivas poderiam ser usadas no desenvolvimento de variedades de plantas transgênicas, pois as proteínas Vip3 não têm semelhança com as Cry e, portanto, reconhecem diferentes receptores no intestino médio

Tabela 2. Suscetibilidade e inibição de desenvolvimento (ID) de larvas neonatas de Chrysodeixis includens às proteínas Cry1 e Vip3 de Bacillus thuringiensis.

\begin{tabular}{ccccc}
\hline Proteína & $\mathrm{b} \pm \mathrm{EP}^{(1)}$ & $\mathrm{CL}_{50}(\mathrm{IC} \text { min-max })^{(2)}$ & $\mathrm{ID}(\%)^{(3)}$ & $\mathrm{CL}_{90}(\mathrm{IC} \text { min-max })^{(4)}$ \\
\hline Cry1Aa & $3,0 \pm 0,5$ & $27(21-33,5)$ & 90 & $71(53-118,5)$ \\
Cry1Ab & $3,9 \pm 0,5$ & $35(27,5-43,7)$ & 65 & $96(71,5-159,6)$ \\
Cry1Ac & $4,7 \pm 1,1$ & $13,3(10-17)$ & 87 & $24,8(19-44,7)$ \\
Cry1Ca & $1,6 \pm 0,3$ & $7,7(4-13)$ & 80 & $48,8(25-232,3)$ \\
Cry1Ea & $3,0 \pm 0,5$ & $17,7(13-23)$ & 65 & $46(33-86,7)$ \\
Vip3Aa & $2,8 \pm 0,5$ & $35,5(27,5-45,5)$ & 83 & $99,2(70,5-193,8)$ \\
Vip3Ae & $2,2 \pm 0,3$ & $80,8(61-110,7)$ & 85 & $301,2(193,5-693)$ \\
Vip3Af & $1,2 \pm 0,3$ & $1,4(0,64-2,5)$ & 70 & $15,5(7-100,7)$ \\
\hline
\end{tabular}

${ }^{(1)}$ Coeficiente angular da reta \pm erro-padrão. ${ }^{(2)} \mathrm{Os}$ valores de $\mathrm{CL}_{50}$ são expressos em ng cm${ }^{-2}$, com $95 \%$ de intervalo de confiança (IC). ${ }^{(3)}$ Inibição do desenvolvimento das larvas alimentadas com as doses de $\mathrm{CL}_{50} .{ }^{\left({ }_{4}\right)} \mathrm{Os}$ valores de $\mathrm{CL}_{90}$ são expressos em ng cm-2 com $95 \%$ de intervalo de confiança (IC). Os valores representam a média de três réplicas de 16 larvas por réplica $(\mathrm{n}=48)$. 
de larvas suscetíveis (Abdelkefi-Mesrati et al., 2011). Assim, algumas combinações destas diferentes proteínas podem atuar em sinergia para o controle de insetos-praga.

Para A. gemmatalis, a combinação Vip3Aa+Cry1Ab apresentou sinergia e foi cerca de quatro a sete vezes mais eficiente do que as combinações Vip3Aa+Cry1Ea e Vip3Ae + Cry1Ab (Tabela 3), que também se apresentaram sinérgicas com os valores de $\mathrm{CL}_{90}$.

$\mathrm{O}$ uso simultâneo de Vip3Aa+Cry1Ea apresentou sinergia no controle de $C$. includens, tendo sido duas vezes mais efetiva do que as combinações Vip3Ae+Cry1Aa e Vip3Ae+Cry1Ea. A combinação Vip3Aa+Cry1Ea foi três vezes mais efetiva do que as combinações Vip3Ae+Cry1Aa e Vip3Ae+Cry1Ea (Tabela 4).

As demais combinações, avaliadas para A. gemmatalis e $C$. includens, apresentaram valores de fator de sinergismo inferior ao requerido para sugerir benefício no controle destas pragas (FS>1). Essas combinações indicaram interações antagônicas, e são necessários estudos para verificar qual o mecanismo de ação delas em cada espécie.

Existem relatos de que proteínas Cry podem agir sinergicamente e aumentar sua toxicidade contra insetos suscetíveis (Wirth et al., 2001; Xue et al., 2005). Esses estudos ajudam na busca de genes cry mais apropriados para introdução no genoma de plantas cultivadas.

A eficácia de uma combinação não é estimada com base na eficácia dos componentes individuais, mas com base no estudo de sinergismo (Bliss, 1939). Plantas piramidadas oferecem alternativa viável para retardar a resistência em populações de insetos-praga. A principal vantagem deste método é que a resistência causada por uma toxina pode não acarretar resistência cruzada à outra toxina (Alyokhin, 2011), uma vez que os receptores são diferentes. Segundo Abdelkefi-Mesrati et al. (2011), as diferenças entre toxinas de $B$. thuringiensis são muito importantes, e o uso de toxinas Vip3 juntamente com as Cry seria indicado para inibir a resistência em insetos-pragas.

Tabela 3. Avaliação de sinergismo, quanto à $\mathrm{CL}_{50}$ e à $\mathrm{CL}_{90}$, de combinações de pró-toxinas Vip3a e Cry1 para Anticarsia gemmatalis.

\begin{tabular}{|c|c|c|c|c|c|c|c|c|}
\hline \multirow{2}{*}{$\begin{array}{l}\text { Combinação } \\
\text { de proteínas }\end{array}$} & \multirow[t]{2}{*}{ Proporção $^{(1)}$} & \multirow[t]{2}{*}{$\mathrm{b} \pm \mathrm{EP}^{(2)}$} & \multicolumn{3}{|c|}{$\mathrm{CL}_{50}\left(\mathrm{ng} \mathrm{cm}^{-2}\right)$} & \multicolumn{3}{|c|}{$\mathrm{CL}_{90}\left(\mathrm{ng} \mathrm{cm}^{-2}\right)$} \\
\hline & & & Observada $\left(\mathrm{IC}_{95}\right)$ & Esperada $^{(3)}$ & $\mathrm{FS}^{(4)}$ & Observada $\left(\mathrm{IC}_{95}\right)$ & Esperada $^{(3)}$ & $\mathrm{FS}^{(4)}$ \\
\hline Vip3Aa+Cry1Aa & $3: 1$ & $2,4 \pm 0,6$ & $26(18,2-37,7)$ & 3,6 & 0,14 & $87,5(54,0-284)$ & 38,3 & 0,44 \\
\hline Vip3Aa+Cry1Ac & $7: 1$ & $1,8 \pm 0,4$ & $39(27-66,4)$ & 3,04 & 0,08 & $188(97,3-791,3)$ & 38,8 & 0,2 \\
\hline Vip3Aa+Cry1Ab & $1: 7$ & $1,2 \pm 0,3$ & $3,8(1,7-6,3)$ & 22,2 & 5,8 & $39,6(19,6-205,1)$ & 178,8 & 4,5 \\
\hline Vip3Aa+Cry1Ca & $1: 4$ & $1,4 \pm 0,3$ & $20(10-37)$ & 14,04 & 0,70 & $167,3(78-856)$ & 82,3 & 0,49 \\
\hline Vip3Aa+Cry1Ea & $1: 10$ & $2,6 \pm 0,6$ & $26,5(17,1-36,2)$ & 29,2 & 1,1 & $80(54,4-185,6)$ & 135,8 & 1,7 \\
\hline Vip3Ae+Cry1Aa & $3: 1$ & $2,6 \pm 0,5$ & $31,3(24-44)$ & 3,5 & 0,11 & $96,3(62,2-244)$ & 41,8 & 0,43 \\
\hline Vip3Ae+Cry1Ac & $7: 1$ & $1,3 \pm 0,3$ & $18,4(11-39,2)$ & 2,9 & 0,16 & $160(62,5-2.006)$ & 41,9 & 0,26 \\
\hline Vip3Ae+Cry1Ab & $1: 8$ & $2,0 \pm 0,3$ & $15,9(11-22,8)$ & 22,5 & 1,4 & $68,8(42,7-162,2)$ & 187,2 & 2,7 \\
\hline Vip3Ae+Cry1Ca & $1: 5$ & $1,7 \pm 0,4$ & $68,3(45-146,2)$ & 14,5 & 0,21 & $367,1(164-3.071)$ & 86,7 & 0,23 \\
\hline Vip3Ae+Cry1Ea & $1: 10$ & $2,7 \pm 0,6$ & $35(25-48)$ & 28,1 & 0,8 & $100,5(68-236)$ & 130,5 & 1,3 \\
\hline Vip3Af+Cry1Aa & $2: 1$ & $1,2 \pm 0,3$ & $25(13-78,4)$ & 2,7 & 0,1 & $266(83-6.851)$ & 6,9 & 0,02 \\
\hline Vip3Af + Cry1Ac & $5: 1$ & $1,0 \pm 0,1$ & $17(9-30,3)$ & 2,2 & 0,13 & $301(135,5-1.111)$ & 7,3 & 0,02 \\
\hline Vip3Af + Cry1 Ab & $1: 11$ & $1,8 \pm 0,4$ & $51,9(34,5-105,8)$ & 21,7 & 0,4 & $258,4(120,4-1.940)$ & 145,7 & 0,56 \\
\hline Vip3Af+Cry1Ca & $1: 7$ & $1,4 \pm 0,3$ & $273,6(172-509,3)$ & 13,8 & 0,05 & $2.198,4(962,8-14.474)$ & 52,3 & 0,02 \\
\hline Vip3Af+Cry1Ea & $1: 15$ & $2,8 \pm 0,5$ & $35(27-46)$ & 28,3 & 0,81 & $100(70,2-191,5)$ & 99,8 & 0,99 \\
\hline
\end{tabular}

${ }^{(1)}$ As proporções de proteínas foram determinadas de acordo com a Tabela $1\left(\mathrm{CL}_{50} \mathrm{e} \mathrm{CL}{ }_{90}\right) .{ }^{\left({ }^{2}\right)}$ Coeficiente angular da reta \pm erro-padrão, ${ }^{(3)}$ Mortalidade esperada. (4)FS, fator de sinergismo calculado como a razão entre a CL esperada e a observada, em que valores maiores do que 1 indicam sinergismo entre as proteínas, valores menores do que 1 indicam antagonismo entre as proteínas, e valores iguais a 1 indicam a ocorrência de toxicidade aditiva. 
Tabela 4. Avaliação de sinergismo, quanto à $\mathrm{CL}_{50}$ e à $\mathrm{CL}_{90}$, de combinações de pró-toxinas Vip3a e Cry1 para Chrysodeixis includens.

\begin{tabular}{|c|c|c|c|c|c|c|c|c|}
\hline \multirow{2}{*}{$\begin{array}{l}\text { Combinação } \\
\text { de proteínas }\end{array}$} & \multirow[t]{2}{*}{ Proporçãa ${ }^{(1)}$} & \multirow[t]{2}{*}{$\mathrm{b} \pm \mathrm{EP}^{(2)}$} & \multicolumn{3}{|c|}{$\mathrm{CL}_{50}\left(\mathrm{ng} \mathrm{cm}^{-2}\right)$} & \multicolumn{3}{|c|}{$\mathrm{CL}_{90}\left(\mathrm{ng} \mathrm{cm}^{-2}\right)$} \\
\hline & & & Observada $\left(\mathrm{IC}_{95}\right)$ & Esperada $^{(3)}$ & $\mathrm{FS}^{(4)}$ & Observada $\left(\mathrm{IC}_{95}\right)$ & Esperada $^{(3)}$ & $\mathrm{FS}^{(4)}$ \\
\hline Vip3Aa+Cry1Aa & $1: 1$ & $1,7 \pm 0,3$ & $33(20,1-50)$ & 30,6 & 0,93 & $173(105-397)$ & 82,6 & 0,5 \\
\hline Vip3Aa+Cry1Ac & $3: 1$ & $2,1 \pm 0,3$ & $53(35,3-76,4)$ & 25 & 0,5 & $218,6(139-465)$ & 62 & 0,28 \\
\hline $\mathrm{Vip} 3 \mathrm{Aa}+\mathrm{Cry} 1 \mathrm{Ab}$ & $1: 1$ & $1,3 \pm 0,2$ & $72,75(41-113)$ & 32,5 & 0,5 & $616(339-1.766)$ & 97,57 & 0,16 \\
\hline Vip3Aa+Cry1Ca & $5: 1$ & $3,5 \pm 0,6$ & $72,3(55,4-94,1)$ & 22,1 & 0,3 & $167,5(123,1-289,2)$ & 73,8 & 0,44 \\
\hline Vip3Aa+Cry1Ea & $2: 1$ & $1,6 \pm 0,2$ & $6,5(4-9,6)$ & 26,5 & 4,1 & $39,3(24,2-87,5)$ & 71,5 & 1,8 \\
\hline Vip3Ae+Cry1Aa & $3: 1$ & $1,6 \pm 0,3$ & $18,9(12,4-29,7)$ & 53,9 & 2,8 & $119,6(61,7-544,5)$ & 182,5 & 1,5 \\
\hline Vip3Ae+Cry1Ac & $6: 1$ & $2,5 \pm 0,6$ & $80,2(57,5-119,2)$ & 46,8 & 0,6 & $259(159,5-819,3)$ & 162,2 & 0,6 \\
\hline Vip3Ae+Cry1Ab & $2: 1$ & $1,3 \pm 0,2$ & $146(91,5-244,5)$ & 56,2 & 0,4 & $1.408(658-6.509)$ & 196,3 & 0,14 \\
\hline Vip3Ae+Cry1Ca & $10: 1$ & $3,9 \pm 0,7$ & $62(51-74)$ & 43,3 & 0,7 & $130(103,5-197)$ & 173,2 & 1,3 \\
\hline Vip3Ae+Cry1Ea & $5: 1$ & $1,7 \pm 0,3$ & $20,3(14-30)$ & 50,62 & 2,5 & $112(67-272)$ & 177,7 & 1,6 \\
\hline Vip3Af+Cry1Aa & $1: 19$ & $2,6 \pm 0,5$ & $21,3(15,3-29,1)$ & 14,1 & 0,66 & $66,4(45-139)$ & 44,4 & 0,67 \\
\hline Vip3Af+Cry1Ac & $1: 10$ & $1,3 \pm 0,2$ & $7,1(3,7-12)$ & 7,5 & 1,05 & $65,5(33-250,3)$ & 20,6 & 0,31 \\
\hline Vip3Af+Cry1Ab & $1: 25$ & $1,3 \pm 0,2$ & $303,8(187-545)$ & 18,2 & 0,06 & $2.662,5(1.183-15.889)$ & 55,11 & 0,02 \\
\hline Vip3Af+Cry1Ca & $1: 6$ & $1,4 \pm 0,2$ & $30,8(19-50)$ & 4,7 & 0,15 & $252,4(133-724)$ & 31,7 & 0,11 \\
\hline Vip3Af+Cry1Ea & $1: 13$ & $2,2 \pm 0,5$ & $15,6(11-24,4)$ & 9,6 & 0,6 & $59(34-250)$ & 30,8 & 0,5 \\
\hline
\end{tabular}

${ }^{(1)}$ As proporções de proteínas foram determinadas de acordo com a Tabela $1\left(\mathrm{CL}_{50}\right.$ e $\left.\mathrm{CL}_{90}\right)$. ${ }^{(2)}$ Coeficiente angular da reta \pm erro-padrão. ${ }^{(3)}$ Mortalidade esperada. ${ }^{(4)} \mathrm{FS}$, fator de sinergismo calculado como a razão entre a CL esperada e a observada, em que valores maiores do que 1 indicam sinergismo entre as proteínas, valores menores do que 1 indicam antagonismo entre as proteínas, e valores iguais a 1 indicam a ocorrência de toxicidade aditiva.

\section{Conclusões}

1. Entre as pró-toxinas avaliadas, Cry1Ac e Vip3Af são as mais efetivas para o controle de Anticarsia gemmatalis e Chrysodeixis includens, como toxinas isoladas.

2. A maior inibição de desenvolvimento é proporcionada por CrylAc e Cry1Ca.

3. A combinação Vip3Aa+Cry1Ea destaca-se e é promissora para a construção de plantas piramidadas, com vistas ao controle de $A$. gemmatalis e $C$. includens.

\section{Agradecimentos}

À Coordenação de Aperfeiçoamento de Pessoal de Nível Superior (Capes), pelo apoio financeiro; ao Prof. Dr. José Roberto Postali Parra e à técnica Neide Graciano Zério, do Laboratório de Biologia dos Insetos, Escola Superior de Agricultura Luiz de Queiroz (Esalq), Universidade de São Paulo (USP), pelo fornecimento de ovos das espécies avaliadas neste estudo; ao Prof. Dr. Ruud de Maagd, por ceder os clones das proteínas
Cry1; e à empresa Bayer CropScience, por ceder os clones das proteínas Vip3A.

\section{Referências}

ABDELKEFI-MESRATI, L.; BOUKEDI, H.; DAMMAK-KARRAY, M.; SELLAMI-BOUDAWARA, T; JAOUA, S.; TOUNSI, S. Study of the Bacillus thuringiensis Vip3Aa16 histopathological effects and determination of its putative binding proteins in the midgut of Spodoptera littoralis. Journal of Invertebrate Pathology, v.106, p.250-254, 2011. DOI: 10.1016/j.jip.2010.10.002.

ALYOKHIN, A. Scant evidence supports EPA's pyramided Bt corn refuge size of 5\%. Nature Biotechnology, v.29, p.577-578, 2011. DOI: $10.1038 /$ nbt.1911

ASHFAQ, M.; YOUNG, S.Y.; MCNEW, R.W. Larval mortality and development of Pseudoplusia includens (Lepidoptera: Noctuidae) reared on a transgenic Bacillus thuringiensis-cotton cultivar expressing Cry1Ac insecticidal protein. Journal of Economic Entomology, v.94, p.1053-1058, 2011. DOI: 10.1603/0022-0493-94.5.1053.

BERGAMASCO, V.B.; MENDES, D.R.P.; FERNANDES, O.A.; DESIDÉRIO, J.A.; LEMOS, M.V.F. Bacillus thuringiensis Cry1Ia10 and Vip3Aa protein interactions and 
their toxicity in Spodoptera spp. (Lepidoptera). Journal of Invertebrate Pathology, v.112, p.152-158, 2013. DOI: 10.1016/j. jip.2012.11.011.

BERNARDI, O.; MALVESTITI, G.S.; DOURADO, P.M.; OLIVEIRA, W.S.; MARTINELLI, S.; BERGER, G.U.; HEAD, G.P.; OMOTO, C. Assessment of the high-dose concept and level of control provided by MON 87701 x MON 89788 soybean against Anticarsia gemmatalis and Pseudoplusia includens (Lepidoptera: Noctuidae) in Brazil. Pest Management Science, v.68, p.1083-1091, 2012. DOI: 10.1002/ps.3271.

BLISS, C.I. The toxicity of poisons applied jointly. Annals of Applied Biology, v.26, p.585-615, 1939. DOI: 10.1111/ j.1744-7348.1939.tb06990.x.

BRAVO, A.; GILL, S.S.; SOBERÓN, M. Mode of action of Bacillus thuringiensis Cry and Cyt toxins and their potential for insect control. Toxicon, v.49, p.423-435, 2007. DOI: 10.1016/j. toxicon.2006.11.022.

BRAVO, A.; SARABIA, S.; LOPEZ, L.; ONTIVEROS, H.; ABARCA, C.; ORTIZ, A.; ORTIZ, M.; LINA, L.; VILLA-LOBOS, FJ.; GUADALUPE, P.; NUÑEZ-VALDEZ, M.E.; SOBERÓN, M.; QUINTERO, R. Characterization of cry genes in Mexican Bacillus thuringiensis strain collection. Applied and Environmental Microbiology, v.64, p.4965-4972, 1998.

CHAKROUN, M.; BEL, Y.; CACCIA, S.; ABDELKEFI-MESRATI, L.; ESCRICHE, B. Susceptibility of Spodoptera frugiperda and S. exigua to Bacillus thuringiensis Vip3Aa insecticidal protein. Journal of Invertebrate Pathology, v.110, p.334-339, 2012. DOI: 10.1016/j.jip.2012.03.021.

COMISSÃO TÉCNICA NACIONAL DE BIOSSEGURANÇA. Parecer técnico $n^{\circ} 1757$, de 19 de março de 2009. 2009. Disponível em: $\quad<$ http://www.ctnbio.gov.br/index.php/content/view/12884. html>. Acesso em: 3 abr. 2014.

COMISSÃO TÉCNICA NACIONAL DE BIOSSEGURANÇA. Parecer técnico $n^{\circ} 2722$, de 18 de novembro de 2010. Liberação comercial de milho geneticamente modificado resistente a insetos e tolerante a herbicidas. 2010. Disponível em: <http:// www.ctnbio.gov.br/index.php/content/view/15727.html>. Acesso em: 3 abr. 2014.

DHURUA, S.; GUJAR, G.T. Field-evolved resistance to Bt toxin CrylAc in the pink bollworm, Pectinophora gossypiela (Saunders) (Lepidoptera: Gelechiidae), from India. Pest Management Science, v.67, p.803-898, 2011. DOI: 10.1002/ps.2127.

FERRÉ, J.; VAN RIE, J. Biochemistry and genetics of insect resistance to Bacillus thuringiensis. Annual Review of Entomology, v.47, p.501-533, 2002. DOI: 10.1146/annurev. ento.47.091201.145234.

FIGUEIREDO, C.S.; MARUCCI, S.C.; TEZZA, R.I.D.; LEMOS, M.V.F.; DESIDÉRIO, J.A. Caracterização do gene vip3A e toxicidade da proteína Vip3Aa50 à lagarta-do-cartucho e à lagartada-soja. Pesquisa Agropecuária Brasileira, v.48, p.1220-1227, 2013. DOI: 10.1590/S0100-204X2013000900005.

FINNEY, D.J. Probit analysis. Cambridge: Cambridge University, 1971. 333p.
FIUZA, L.M.; KNAAK, N.; SILVA, R.F.P. da; HENRIQUE, J.A.P. Receptors and lethal effect of Bacillus thuringiensis insecticidal crystal proteins to the Anticarsia gemmatalis (Lepidoptera, Noctuidae). ISRN Microbiology, v.2013, 7p., 2013. DOI: $10.1155 / 2013 / 940284$

HERRERO, S.; GONZALEZ-CABRERA, J.; FERRÉ, J.; BAKKER, P.L.; DE MAAGD, R.A. Mutations in the Bacillus thuringiensis CrylCa toxin demonstrate the role of domains II and III in specificity towards Spodoptera exigua larvae. Biochemical Journal, v.384, p.507-513, 2004. DOI: 10.1042/BJ20041094.

HUANG, F.; LEONARD, B.R.; GABLE, R.H. Comparative susceptibility of European corn borer, Southwestern corn borer, and sugarcane borer (Lepidoptera: Crambidae) to Cry1 Ab protein in a commercial Bacillus thuringiensis corn hybrid. Journal of Economic Entomology, v.99, p.194-202, 2006. DOI: 10.1603/0022-0493(2006)099[0194:CSOECB]2.0.CO;2.

JAMES, C. Global status of commercialized biotech/GM crops: 2011. Ithaca: International Service for the Acquisition of Agri-Biotech Applications, 2011. (ISAAA Brief, 43).

JAMES, C. Global status of commercialized transgenic crops: 2002. Ithaca: International Service for the Acquisition of Agri-Biotech Applications, 2002. 24p. (ISAAA Brief, 27).

LAEMMLI, U.K. Cleavage of structural proteins during assembly of the head of bacteriophage T4. Nature, v.227, p.680-685, 1970. DOI: $10.1038 / 227680 \mathrm{a} 0$.

LEE, M.K.; WALTERS, F.S.; HART, H.; PALEKAR, N.; CHEN, J.-S. The mode of action of the Bacillus thuringiensis vegetative insecticidal protein Vip3A differs from that of Cry1 Ab delta-endotoxin. Applied and Environmental Microbiology, v.69, p.4648-4657, 2003. DOI: 10.1128/AEM.69.8.4648-4657.2003.

LIGHTWOOD, D.J.; ELLAR, D.J.; JARRETT, P. Role of proteolysis in determining potency of Bacillus thuringiensis Cry1 Ac delta-endotoxin. Applied and Environmental Microbiology, v.66, p.5174-5181, 2000. DOI: 10.1128/AEM.66.12.5174-5181.2000.

LOGUERCIO, L.L.; BARRETO M.L.; ROCHA T.L.; SANTOS C.G.; TEIXEIRA F.F.; PAIVA E. Combined analysis of supernatant-based feeding bioassays and PCR as a first-tier screening strategy for Vip-derived activities in Bacillus thuringiensis strains effective against tropical fall armyworm. Journal of Applied Microbiology, v.93, p.269-277, 2002. DOI: 10.1046/j.1365-2672.2002.01694.X.

MONNERAT, R.G.; BATISTA, A.C.; MEDEIROS, P.T. de; MARTINS, E.S.; MELATTI, V.M.; PRAÇA, L.B.; DUMAS, V.F.; MORINAGA, C.; DEMO, C.; GOMES, A.C.M.; FALCÃO, R.; SIQUEIRA, C.B.; SILVA-WERNECK, J.O.; BERRY, C. Screening of Brazilian Bacillus thuringiensis isolates active against Spodoptera frugiperda, Plutella xylostella and Anticarsia gemmatalis. Biological Control, v.41, p.291-295, 2007. DOI: 10.1016/j.biocontrol.2006.11.008.

NATIONAL CENTER FOR BIOTECHNOLOGY INFORMATION. Isp3a protein [Bacillus thuringiensis]: GenBank: CAI43275.1. 2013a. Available at: <https://www.ncbi. nlm.nih.gov/protein/CAI43275>. Accessed on: 28 Nov. 2013.

NATIONAL CENTER FOR BIOTECHNOLOGY INFORMATION. Isp3c protein [Bacillus thuringiensis]: 
GenBank: CAI43277.1. 2013b. Available at: <https://www.ncbi. nlm.nih.gov/protein/CAI43277>. Accessed on: 28 Nov. 2013.

NATIONAL CENTER FOR BIOTECHNOLOGY INFORMATION. vip3A(a) [Bacillus thuringiensis]: GenBank: AAC37036.1. 2013C. Available at: <https:/www.ncbi.nlm.nih. gov/protein/AAC37036>. Accessed on: 28 Nov. 2013.

STEWART JUNIOR, C.N.; ADANG, M.J.; ALL, J.N.; BOERMA, R.; CARDINEAU, G.; TUCKER, D.; PARROT, W.A. Genetic transformation, recovery, and characterization for a fertile soybean transgenic for a synthetic Bacillus thuringiensis cryAc gene. Plant Physiology, v.112, p.121-129, 1996. DOI: 10.1104/pp.112.1.121.

STORER, N.P.; BABCOCK, J.M.; SCHLENZ, M.; MEADE, T.; THOMPSON, G.D.; BING, J.W.; HUCKABA, R.M. Discovery and characterization of field resistance to Bt Maize: Spodoptera frugiperda (Lepidoptera: Noctuidae) in Puerto Rico. Journal of Economic Entomology, v.103, p.1031-1038, 2010. DOI: 10.1603/ EC10040.

TABASHNIK, B.E. Evaluation of synergism among Bacillus thuringiensis toxins. Applied and Environmental Microbiology, v.58, p.3343-3346, 1992.

TABASHNIK, B.E.; LIU, Y.B.; MAAGD, R.A. de; DENNEHY, T.J. Cross-resistance of pink bollworm (Pectinophora gossypiella) to Bacillus thuringiensis toxins. Applied and Environmental Microbiology, v.66, p.4582-4584, 2000. DOI: 10.1128/ AEM.66.10.4582-4584.2000.

VAN RENSBURG, J.B.J. First report of field resistance by the stem borer Busseola fusca (Fuller) to Bt transgenic maize. South African Journal of Plant and Soil, v.24, p.147-151, 2007. DOI: 10.1080/02571862.2007.10634798.

WALKER, D.R.; ALL, J.N.; MCPHERSON, R.M.; BOERMA, H.R.; PARROTT, W.A. Field evaluation of soybean engineered with a synthetic $c r y l A c$ transgene for resistance to corn earworm, soybean looper, velvetbean caterpillar (Lepidoptera, Noctuidae), and lesser cornstalk borer (Lepidoptera, Pyralidae). Journal of Economic Entomology, v.93, p.613-622, 2000. DOI: 10.1603/0022-0493-93.3.613.

WIRTH, M.C.; DELÉClUSE, A.; WALTON, W.E. Cyt1Ab and CyteBa1 from Bacillus thuringiensis subsp. medellin and $B$. thuringiensis subsp. israelensis synergize Bacillus sphaericus against Aedes aegypti and resistant Culex quinquefasciatus (Diptera: Culicidae). Applied and Environmental Microbiology, v.67, p.3280-3284, 2001. DOI: 10.1128/AEM.67.7.32803284.2001 .

WU, D.; JOHNSON, J.J.; FEDERECI, B.A. Synergism of mosquitocidal toxicity between CytA and CryIVD proteins using inclusions produced from cloned genes of Bacillus thuringiensis. Molecular Microbiology, v.13, p.965-972, 1994. DOI: 10.1111/ j.1365-2958.1994.tb00488.x.

XUE, J.-L.; CAI, Q.-X.; ZHENG, D.-S.; YUAN, Z.M. The synergistic activity between Cry1Aa and Cry1C from Bacillus thuringiensis against Spodoptera exigua and Helicoverpa armigera. Letters in Applied Microbiology, v.40, p.460-465, 2005. DOI: 10.1111/j.1472-765X.2005.01712.x.

YU, C.G.; MULLINS, M.A.; WARREN, G.W.; KOZIEL, M.G.; ESTRUCH, J.J. The Bacillus thuringiensis vegetative insecticidal protein Vip3A lyses midgut ephitelium cells of susceptible insects. Applied and Environmental Microbiology, v.63, p.532-536, 1997.

ZHANG, L.; HUANG, F.; LEONARD, B.R.; CHEN, M.; CLARK, T.; ZHU, Y.C.; WANGLIA, D.S.; YANG, F.; NIU, Y. Susceptibility of Cry1Ab maize-resistant and -susceptible strains of sugarcane borer (Lepidoptera: Crambidae) to four individual Cry proteins. Journal of Invertebrate Pathology, v.112, p.267-272, 2013. DOI: 10.1016/j.jip.2012.12.007.

$\overline{\text { Recebido em } 28 \text { de novembro de } 2013 \text { e aprovado em } 31 \text { de janeiro de } 2014}$ 